Ann. Génét. Sél. anim., 1972, 4 (3), 477-480.

NOTE

\title{
PARAMÈTRES GÉNÉTIQUES \\ DE LA GROISSANCE DES CHEVRETTES SAANEN EN STATION DE TESTAGE
}

\author{
G. RICORDEAU, B. POUJARDIEU et J. BOUILLON * \\ avec la collaboration technique de $A$. LAJous \\ Laboratoive de Génétique des Petits Ruminants, \\ Laboratoire de Méthodologie génétique, \\ Centre de Recherches de Toulouse, I. N.R. A., \\ BP 12, 31 - Castanet-Tolosan \\ * Station de Testage caprin, \\ 48 - Moissac
}

\section{RÉSUMÉ}

Cette étude a été effectuée sur I o8o chevrettes de race Saanen, issues de 64 boucs et pesées régulièrement jusqu'à 8 mois. Les paramètres génétiques ont été calculés sur les données transformées en probit pour éliminer l'effet significatif de l'année.

L'héritabilité passe de 0,63 pour le poids à I mois, à 0,50 en moyenne pour les poids à 2, 3,5 et 7 mois. Les corrélations génétiques, entre le poids à 3 mois d'une part et les poids à 5 et 7 mois d'autre part, sont respectivement de 0,91 et 0,89 ; elles montrent tout l'intérêt du poids à 3 mois en vue de la sélection des chevrettes destinées à la reproduction.

\section{INTRODUCTION}

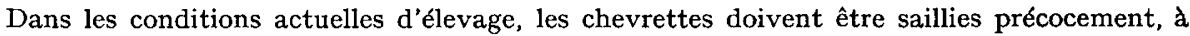
7-8 mois, a fin d'entrer en production à $\mathrm{r}$ an. Pour obtenir des résultats satisfaisants, il est cependant nécessaire que les jeunes reproductrices atteignent un poids moyen de $32 \mathrm{~kg}$ à la saillie (FEHR, I97I).

Cette note a pour but d'estimer les paramètres génétiques de la croissance enregistrée de I à 7 mois : elle complète l'étude précédente concernant l'effet du gène sans cornes $\mathrm{P}$ sur le poids des chevrettes d'élevage (Ricordeau, Poujardieu et Bouilion, i972). 


\title{
MATÉRIEL E'T MÉTHODES
}

\author{
Effectifs
}

Ces résultats ont été obtenus de 1965 à I970, à la Station de testage caprin de Moissac - station de contrôle de descendance - sur I o8o chevrettes de race Saanen, pesées régulièrement pendant au moins 8 mois. Ces chevrettes sont issues de 64 boucs. Par suite de la prolificité des mères accouplées aux boucs à tester - près de 2 produits par mise bas - chaque lot de filles, d'un effectif moyen de 16 à I 7, compte des demi-sœurs et des pleine-sœurs : de ce fait, le coefficient de parenté par descendance est en moyenne de $0,34^{2}$.

\section{Conditions d'élevage}

Les chevrettes sont achetées aux éleveurs (de janvier à avril) quelques jours après leur naissance et élevées ensuite dans des conditions comparables : lait de chèvre jusqu'à 25 jours environ; aliment d'allaitement jusqu'à 3 mois environ; concentré dès l'âge de 3 semaines limité à $400 \mathrm{~g}$ par jour et fourrage d'excellente qualité à volonté. La stabulation intégrale a été pratiquée les 5 premières années.

\section{Pesées}

Les chevrettes sont pesées une fois tous les mois jusqu'à 8 mois. Les poids à âge-type sont calculés à $\mathbf{I}, 2,3,5$ et 7 mois par intrapolation linéaire, ainsi que les gains moyens journaliers de $I$ à 3 mois et de 3 à 7 mois.

\section{Paramètres génétiques}

Du fait des différences significatives entre années (cf. RicoRDEAU et al., 1972), nous avons effectué 2 transformations pour les 7 variables : transformation en p. Ioo des moyennes annuelles de chaque série de testage et transformation probit à partir des moyennes annuelles.

Les valeurs du coefficient d'héritabilité et de corrélation génétique, de chacun des caractères ou des caractères entre eux, sont déduites de l'analyse de la variance à une voie, des performances des sœurs et demi-sœurs de père, compte tenu du coefficient de parenté moyen par descendance.

\section{RÉSULTATS}

Les valeurs obtenues à partir des 2 transformations sont presque identiques; aussi retiendrons-nous uniquement les résultats correspondants aux variables transformées probit (tabl. r).

Le coefficient d'héritabilité passe de 0,63 pour le poids à un mois, à 0,50 en moyenne pour les poids à 2, 3, 5 et 7 mois. L'héritabilité du gain moyen journalier de I à 3 mois est bonne $(0,5 \mathrm{I})$, mais nettement plus faible de 3 à 7 mois $(0,23)$.

Les valeurs des coefficients de corrélation génétique entre le poids à 7 mois d'une part, les poids à $I, 2$ et 3 mois ou les 2 gains d'autre part, sont élevées. En revanche, le poids à I mois est en corrélation génétique négative avec les 2 gains.

La corrélation génétique élevée entre le poids à 3 mois d'une part, les poids à 5 et 7 mois d'autre part $(0,9 \mathbf{I}$ et 0,89$)$, montre tout l'intérêt du poids à 3 mois pour avoir une idée assez précoce du poids moyen des descendances ou pour réaliser une première sélection des chevrettes destinées à la reproduction. 
CROISSANCE DES CHEVRETTES "SAANEN 》

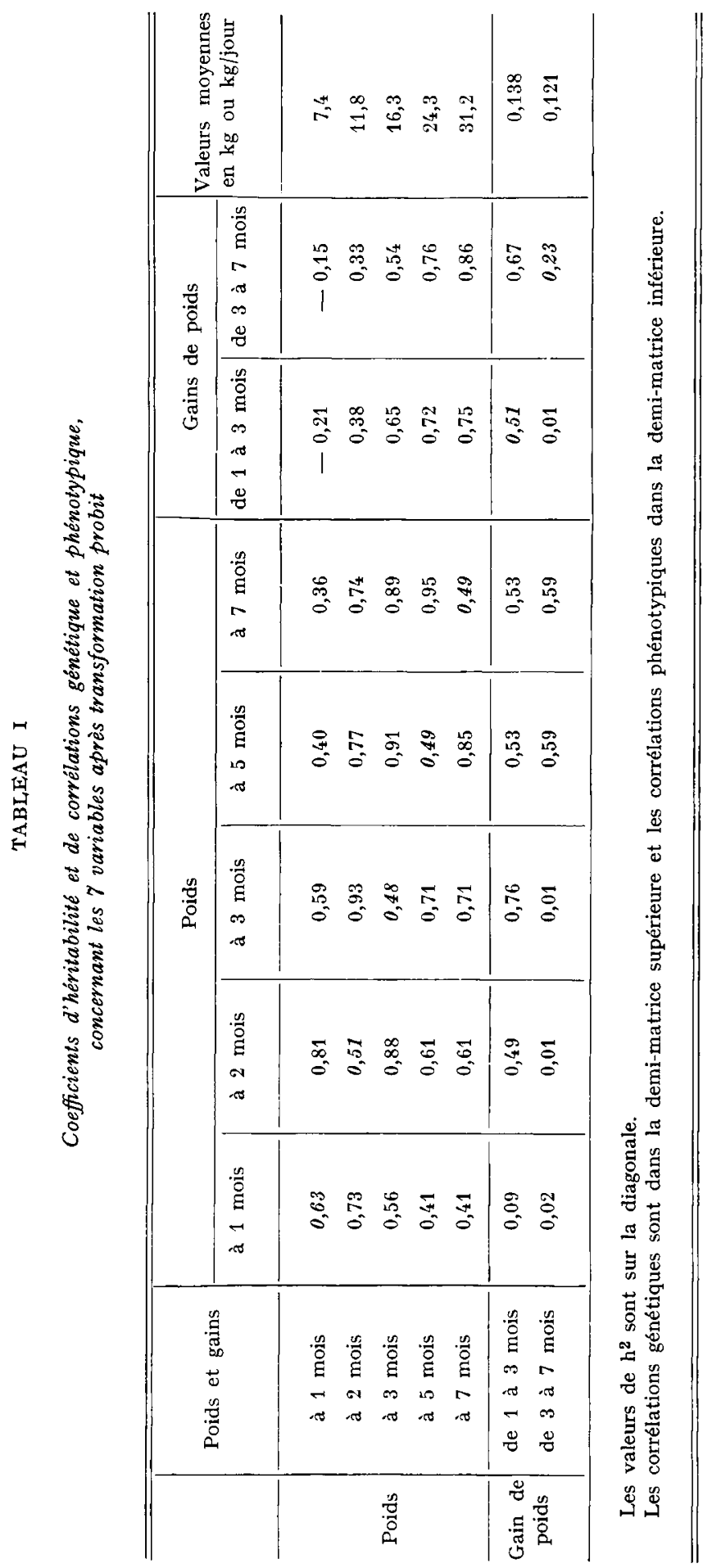




\section{SUMMARY \\ GENETIC PARAMETERS OF GROWTH \\ IN YOUNG SAANEN SHE-GOATS IN A TESTING STATION}

I o8o young Saanen she-goats, the progeny of 64 he-goats, were studied and weighed regularly until 8 months of age.

The genetic limits were computed from data transformed to eliminate the significant effect of the year.

Weight heritability at $\mathrm{I}$ month ranges from 0.63 to an average of 0.50 for weight at $2,3,5$ and 7 months. Genetic correlations between weight at 3 months and weight at 5 and 7 months are 0.91 and 0.89 , respectively. These correlations show that weight at 3 months is valuable as a selection criterium for young breeder she-goats.

\section{RÉFÉRENCES BIBLIOGRAPHIQUES}

FeHr P. M., 1971. Production et alimentation des chevrettes et des chevreaux. Rev. Élevage, 11, II3-I25.

Ricordeau G., Poujardieu B., Bouillon J., 1972. Effet du gène sans cornes P sur le poids des chevrettes d'élevage. Ann. Génét. Sél. anim. 4, 29-34 